\title{
An anti-HIV-1 compound that increases steady-state expression of apoplipoprotein B mRNA-editing enzyme-catalytic polypeptide-like 3G
}

\author{
TOMOHIKO EJIMA ${ }^{1}$, MAYUKO HIROTA ${ }^{1}$, TAMIO MIZUKAMI $^{2}$, MASAMI OTSUKA $^{1}$ and MIKAKO FUJTA $^{3}$ \\ ${ }^{1}$ Department of Bioorganic Medicinal Chemistry, Faculty of Life Sciences, Kumamoto University, Kumamoto 862-0973; \\ ${ }^{2}$ Graduate School of Bioscience, Nagahama Institute of Bio-Science and Technology, Nagahama 526-0829; \\ ${ }^{3}$ Research Institute for Drug Discovery, School of Pharmacy, Kumamoto University, Kumamoto 862-0973, Japan
}

Received May 19, 2011; Accepted June 17, 2011

DOI: $10.3892 /$ ijmm.2011.737

\begin{abstract}
Human apoplipoprotein B mRNA-editing enzymecatalytic polypeptide-like (APOBEC) $3 \mathrm{G}$ (A3G) is an antiviral protein that blocks HIV-1 replication. However, the antiviral activity of A3G is overcome by the HIV-1 protein Vif. This inhibitory function of Vif is related to its ability to degrade $\mathrm{A} 3 \mathrm{G}$ in the proteasome. This finding prompted us to examine the activities of 4-(dimethylamino)-2,6-bis[(N-(2-[(2-nitrophenyl)dithio]ethyl)amino)methyl]pyridine (SN-2) and SN-3. We found that $5 \mu \mathrm{M} \mathrm{SN}-2$ increases the expression of $\mathrm{A} 3 \mathrm{G}$ to a level much higher than that observed in the absence of Vif, without affecting the level of Vif expression. The proteasome inhibitor MG-132 increased the level of both A3G and Vif expression. These results demonstrate that $\mathrm{A} 3 \mathrm{G}$ is ubiquitinated and degraded in the proteasome by a factor other than Vif, and that SN-2 selectively inhibits these processes. Furthermore, $5 \mu \mathrm{M} \mathrm{SN}-2$ significantly inhibited the MAGI cell infectivity of wild-type HIV-1. These findings may contribute to the development of a novel anti-HIV-1 drug.
\end{abstract}

\section{Introduction}

Acquired immunodeficiency syndrome (AIDS), caused by the human immunodeficiency virus type 1 (HIV-1), is a severe disease that is often fatal when patients fail to receive optimal treatment. Clinically approved anti-HIV drugs target several key proteins involved in the HIV life cycle, such as reverse transcriptase, protease, envelope protein, CCR5, and integrase $(1,2)$. When used in combination, these diverse anti-HIV drugs become a highly active antiretroviral therapy (HAART) for

Correspondence to: Dr Mikako Fujita, Research Institute for Drug Discovery, School of Pharmacy, Kumamoto University, 5-1 Oe-honmachi, Kumamoto 862-0973, Japan

E-mail: mfujita@kumamoto-u.ac.jp

Key words: human immunodeficiency virus type 1, apoplipoprotein B mRNA-editing enzyme-catalytic polypeptide-like $3 \mathrm{G}$, Vif, proteasome, disulfide suppression of HIV replication within the body of a patient. However, HIV may easily acquire drug resistance through mutation, and long-term usage of the same anti-HIV drugs leads to side effects. Therefore, new anti-HIV drugs based on novel mechanisms of action are needed.

Human apoplipoprotein B mRNA-editing enzymecatalytic polypeptide-like (APOBEC) 3G (A3G) is a cytidine deaminase which edits DNA. Sheehy et al (3) found that A3G acts as an antiviral factor that blocks HIV-1 replication in the absence of the viral protein Vif. Vigorous studies by a number of groups have revealed that in the absence of Vif, A3G is incorporated into HIV-1 particles. When viral RNA is reverse transcribed in target cells, A3G induces extensive $\mathrm{C} \rightarrow \mathrm{U}$ mutations in the synthesized cDNA, and also physically blocks reverse transcription, rendering the virus noninfectious $(4,5)$. In contrast, the presence of Vif reduces the amount of virionassociated A3G through proteasome degradation and other degradation-independent mechanisms $(5,6)$. Thus, A3G and Vif are promising targets for the development of new antiHIV drugs.

Recent reports have described several small molecules that inhibit HIV-1 infectivity by degrading Vif in an A3G-dependent manner (7) or suppressing the Vif/A3G interaction $(8,9)$. In particular, the inhibitor TPEN removes zinc from the zinc binding motif of Vif that contributes to the interaction with A3G (9). This finding prompted us to examine the activity of the zinc chelator SN-1, developed as an inhibitor of the zinc finger protein HIV-EP1, and its related compounds 4-(dimethylamino)-2,6-bis[(N-(2-[(2-nitrophenyl)dithio]ethyl) amino)methyl]pyridine (SN-2) and SN-3 (10,11) (Fig. 1). Here, we report on the unexpected activity of disulfide $\mathrm{SN}-2$, which dramatically and selectively increases the expression of $\mathrm{A} 3 \mathrm{G}$ and shows anti-HIV-1 activity.

\section{Materials and methods}

Chemicals. SN-2 was synthesized as described previously (10). Reduction of SN-2 by DTT, followed by aerobic oxidation produced $\mathrm{SN}-3$, a mixture of monomeric and dimeric disulfides as determined by mass spectrometry, with a $65 \%$ yield (Fig. 1). MG-132 and bis(2-nitrophenyl)disulfide were 
<smiles>CN(C)c1cc(CNCCSSc2ccccc2[N+](=O)[O-])nc(CNCCSSc2ccccc2[N+](=O)[O-])c1</smiles>

SN-2

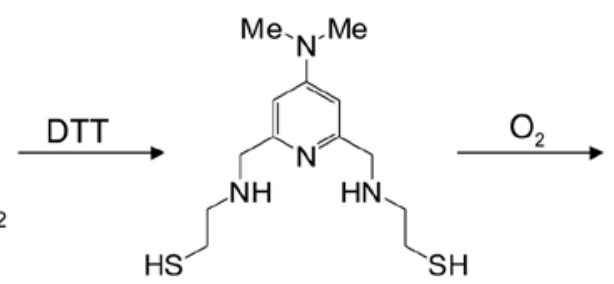

SN-1<smiles>CSCCNCC1CC(N(C)C)=NC(CNCCS)C1</smiles>

SN-3

$\mathrm{n}=1$ or 2

Figure 1. Structure and interconversion of SN compounds.

A

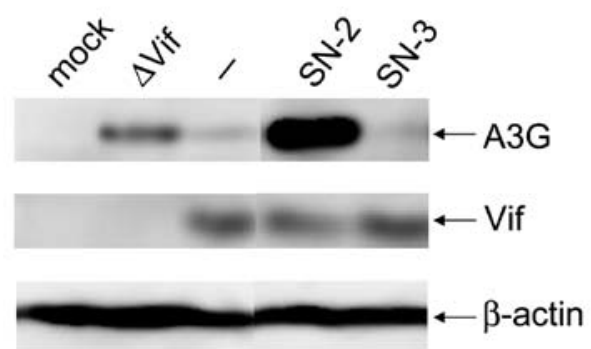

B

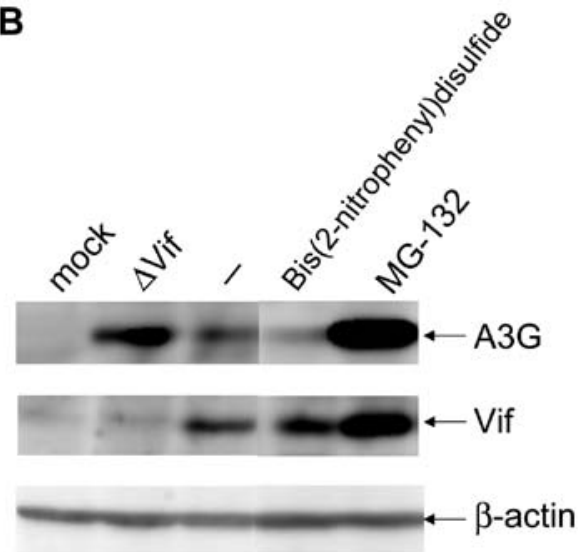

Figure 2. Steady-state expression of A3G and Vif in the presence of $5 \mu \mathrm{M}$ (A) SN-2 or SN-3, and (B) bis(2-nitrophenyl)disulfide or MG-132. Cells (293T) were co-transfected with $1.25 \mu \mathrm{g}$ of pcDNA-APO3G, $6.25 \mu \mathrm{g}$ of pNL-ASCF-fWT, and $2.50 \mu \mathrm{g}$ of pNL-Nd. The designated compound was added to the medium when it was changed at $6 \mathrm{~h}$ post-transfection, and cells were incubated at $37^{\circ} \mathrm{C}$ for an additional $42 \mathrm{~h}$. Cells were lysed in Laemmli sample buffer for Western blot analysis. The antibodies used here were anti-myc (for A3G), monoclonal anti-FLAG M2 (for Vif), or anti- $\beta$-actin clone AC-15 mouse ascites fluid (for $\beta$-actin). Mock, pcDNA3.1(-), pNL-A1 Vif(-) and pNL-Nd; $\Delta$ Vif, pcDNA-APO3G, pNL-A1 Vif(-) and pNL-Nd; dash, no compound.

purchased from Calbiochem Ltd. (San Diego, CA, USA) and Sigma-Aldrich Ltd. (St. Louis, MO, USA), respectively.

Plasmids. A full-length molecular clone pNL4-3 (GenBank accession no. AF324493) (12) was used for production of wild-type (WT) infectious virus. A frame-shift mutant of pNL4-3 designated pNL-Nd $(12,13)$ was used for production of the Vif-minus virus. The subgenomic vector pNL-ASCFfWT (14) was used as an expression vector of Vif carrying a FLAG tag. Vector pcDNA-APO3G (15) was used for expression of A3G carrying a myc tag. pUC19, pNL-A1 Vif(-) (16) and pcDNA3.1(-) (Invitrogen, Carlsbad, CA, USA) were used as mock vectors of pNL4-3, pNL-ASCF-fWT and pcDNAAPO3G, respectively.

Cells. The cell line 293T (17) was cultured in Dulbecco's modified Eagle's medium supplemented with $10 \%$ heat-inactivated fetal bovine serum (FBS). MAGI cells (18) were cultured similarly to $293 \mathrm{~T}$ cells in the presence of $0.2 \mathrm{mg} / \mathrm{ml}$ of G418 and $0.1 \mathrm{mg} / \mathrm{ml}$ of hygromycin $\mathrm{B}$.

Transfection, reverse transcriptase (RT) assay and MAGI assay. The $293 \mathrm{~T}$ cells were transfected by the calcium-phosphate co-precipitation method, as previously described (12). Virus production in the culture supernatants was monitored by RT activity as previously described (19). To determine the infectivity of virions, MAGI assay was performed as previously described (18).

Western blotting. Cell lysates for Western blot analysis were prepared in Laemmli's sample buffer (20) as previously described. Samples were resolved by SDS-PAGE followed by electrophoretic transfer to polyvinylidene fluoride membranes. The membranes were treated with anti-myc antibody (Ab) (Invitrogen), anti-FLAG M2 monoclonal Ab (Sigma-Aldrich), or monoclonal anti- $\beta$-actin clone AC-15 mouse ascites fluid (Sigma-Aldrich). Proteins were visualized using the ECL Plus Western blotting detection reagents (Amersham Biosciences, Buckinghamshire, UK) or an ECL advance Western blotting detection kit (Amersham Biosciences).

\section{Results and Discussion}

We examined the effect of disulfides SN-2 and SN-3 on the steady-state expression of $\mathrm{A} 3 \mathrm{G}$ in the presence of Vif using 293T cells. Cells were co-transfected with pcDNAAPO3G (A3G expression vector carrying a myc tag) (15), pNL-ASCF-fWT (subgenomic vector for expression of Vif and carrying a FLAG tag) (14) and pNL-Nd (Vif defective mutant of HIV-1 infectious clone pNL4-3) $(12,13)$. SN-2 or SN-3 $(5 \mu \mathrm{M})$ were added to the medium when it was changed at $6 \mathrm{~h}$ post-transfection, and the cells were incubated for an 


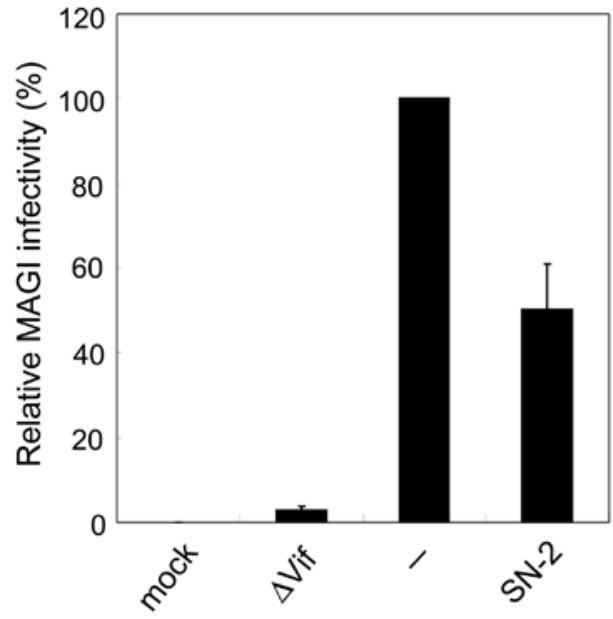

Figure 3. Relative MAGI infectivity in the presence of $5 \mu \mathrm{M} \mathrm{SN}-2$. Cells (293T) were co-transfected with 9.0 $\mu \mathrm{g}$ of pNL4-3 and 1.0 $\mu \mathrm{g}$ of pcDNAAPO3G. SN-2 $(5 \mu \mathrm{M})$ was added to the medium when it was changed at $6 \mathrm{~h}$ post-transfection, and cells were incubated at $37^{\circ} \mathrm{C}$ for an additional $43 \mathrm{~h}$ Supernatants were collected, and MAGI and RT assays were conducted. MAGI activity was normalized to the RT activity to calculate the relative MAGI infectivity. Mock, pUC19 and pcDNA-APO3G; $\Delta$ Vif, pNL-Nd and pcDNA-APO3G; dash, no compound.

additional 42 h. Lysates of vector-transfected 293T cells were prepared using Laemmli's sample buffer (20), and were resolved by SDS-PAGE. Resolved proteins were analyzed by Western immunoblotting using anti-myc, anti-FLAG and anti- $\beta$-actin antibodies.

The steady-state expression of $\mathrm{A} 3 \mathrm{G}$ and Vif is shown in Fig. 2A. In the absence of Vif, a small amount of A3G was detected. In the presence of Vif, the level of A3G expression decreased, as has been previously reported (5). When $5 \mu \mathrm{M}$ $\mathrm{SN}-2$ was present in addition to Vif, A3G expression increased markedly, reaching a level considerably higher than that observed in the absence of Vif. Conversely, SN-3 at $5 \mu \mathrm{M}$ had no effect on the level of $\mathrm{A} 3 \mathrm{G}$ expression. In addition, $\mathrm{SN}-2$ and SN-3 had no effect on the level of Vif.

A possible mechanism giving rise to the marked increase in $\mathrm{A} 3 \mathrm{G}$ expression in the presence of $\mathrm{SN}-2$ could be that A $3 \mathrm{G}$ would normally be degraded by a factor other than Vif in this experimental system, and that the degradation was inhibited by $\mathrm{SN}-2$. We hypothesized that $\mathrm{A} 3 \mathrm{G}$ degradation is proteasome-mediated. Therefore, we examined the effect of the proteasome inhibitor MG-132 on the level of A3G expression in the presence of Vif. The experiment involved using $5 \mu \mathrm{M}$ MG-132 instead of SN-2 or SN-3. As shown in Fig. 2B, MG-132 increased the level of $\mathrm{A} 3 \mathrm{G}$ expression to near that observed with SN-2. Thus, it is presumed that a factor other than Vif also induces proteasome-degradation of $\mathrm{A} 3 \mathrm{G}$.

Notably, MG-132 increased expression of not only A3G, but also Vif. This agrees with previous reports showing that Vif is degraded in the proteasome $(21,22)$. On the contrary, SN-2 did not increase Vif expression (Fig. 2A). It is unlikely that $\mathrm{SN}-2$ inhibits proteasome-degradation of $\mathrm{A} 3 \mathrm{G}$ without inhibiting that of Vif, since proteasome-degradation is a process that uniformly degrades all polyubiquitinated proteins. Most possibly $\mathrm{SN}-2$ targets ubiquitination of $\mathrm{A} 3 \mathrm{G}$.

We also examined the effect on $\mathrm{A} 3 \mathrm{G}$ expression of bis(2-nitrophenyl)disulfide released from SN-2 by disulfide exchange. As shown in Fig. 2B, this compound (5 $\mu \mathrm{M})$, unlike $\mathrm{SN}-2$, did not affect the level of A3G expression. Thus, the activity of $\mathrm{SN}-2$ may require the complete, intact structure.

Finally, since A3G has anti-HIV-1 activity, we examined the anti-HIV-1 activity of SN-2 that increases the amount of A3G. Cells (293T) were co-transfected with pNL4-3 (12) and pcDNA-APO3G (15). We used pNL-Nd (13) as the Vif-defective mutant. When the medium was changed at $6 \mathrm{~h}$ post-transfection, $5 \mu \mathrm{M} \mathrm{SN}-2$ was added to the culture medium, and the cells were incubated for an additional $43 \mathrm{~h}$. Virus production in the culture supernatants was monitored by an RT assay (19). Infectivity of virions was determined using a MAGI assay (18). MAGI activity was normalized to RT activity to calculate relative values.

The results of MAGI infectivity are shown in Fig. 3. The relative MAGI infectivity of WT HIV-1 was halved in the presence of $5 \mu \mathrm{M} \mathrm{SN}-2$. However, the relative MAGI infectivity of SN-2 was higher than that observed in the absence of Vif, whereas expression of $\mathrm{A} 3 \mathrm{G}$ with $\mathrm{SN}-2$ was much higher than in the absence of Vif (Fig. 2A). The reason for this discrepancy remains elusive.

In conclusion, we found that the compound SN-2 dramatically and selectively increases the level of A3G expression. Our results also demonstrate that SN-2 has anti-HIV-1 activity. Although the action mechanism of $\mathrm{SN}-2$ requires further investigation, the findings shown here may contribute to the development of a novel anti-HIV-1 drug.

\section{Acknowledgements}

This study was supported by a Grant-in-Aid for Exploratory Research (no. 22659024) from the Japan Society for the Promotion of Science.

\section{References}

1. Broder S: The development of antiretroviral therapy and its impact on the HIV-1/AIDS pandemic. Antiviral Res 85: 1-18, 2010.

2. Esté JA and Cihlar T: Current status and challenges of antiretroviral research and therapy. Antiviral Res 85: 25-33, 2010.

3. Sheehy AM, Gaddis NC, Choi JD and Malim MH: Isolation of a human gene that inhibits HIV-1 infection and is suppressed by the viral Vif protein. Nature 418: 646-650, 2002.

4. Bishop KN, Verma M, Kim EY, Wolinsky SM and Malim MH: APOBEC3G inhibits elongation of HIV-1 reverse transcripts. PLoS Pathog 4: e1000231, 2008.

5. Goila-Gaur R and Strebel K: HIV-1 Vif, APOBEC, and intrinsic immunity. Retrovirology 5: 51, 2008.

6. Iwatani Y, Chan DSB, Liu L, Yoshii H, Shibata J, Yamamoto N, Levin JG, Gronenborn AM and Sugiura W: HIV-1 Vif-mediated ubiquitination/degradation of APOBEC3G involves four critical lysine residues in its C-terminal domain. Proc Natl Acad Sci USA 106: 19539-19544, 2009.

7. Nathans R, Cao H, Sharova N, Ali A, Sharkey M, Stranska R, Stevenson M and Rana TM: Small molecule inhibition of HIV-1 Vif. Nat Biotechnol 26: 1187-1192, 2008.

8. Cen S, Peng ZG, Li XY, Li ZR, Ma J, Wang YM, Fan B, You XF, Wang YP, Liu F, Shao RG, Zhao LX, Yu L and Jiang JD: Small molecular compounds inhibit HIV-1 replication through specifically stabilizing APOBEC3G. J Biol Chem 285: 16546-16552, 2010.

9. Xiao Z, Ehrlich E, Luo K, Xiong Y and Yu XF: Zinc chelation inhibits HIV Vif activity and liberates antiviral function of the cytidine deaminase APOBEC3G. FASEB J 21: 217-222, 2007.

10. Fujita M, Otsuka M and Sugiura Y: Metal-chelating inhibitors of a zinc finger protein HIV-EP1. Remarkable potentiation of inhibitory activity by introduction of SH groups. J Med Chem 39: 503-507, 1996. 
11. Otsuka M, Fujita M, Sugiura Y, Yamamoto T, Inoue J, Maekawa T and Ishii S: Synthetic inhibitors of regulatory proteins involved in the signaling pathway of the replication of human immunodeficiency virus 1. Bioorg Med Chem 5: 205-215, 1997.

12. Adachi A, Gendelman HE, Koenig S, Folks T, Willey R, Rabson A and Martin MA: Production of acquired immunodeficiency syndrome-associated retrovirus in human and nonhuman cells transfected with an infectious molecular clone. J Virol 59: 284-291, 1986.

13. Adachi A, Ono N, Sakai H, Ogawa K, Shibata R, Kiyomatsu T, Masuike $\mathrm{H}$ and Ueda S: Generation and characterization of the human immunodeficiency virus type 1 mutants. Arch Virol 117: 45-58, 1991.

14. Khamsri B, Fujita M, Kamada K, Piroozmand A, Yamashita T, Uchiyama T and Adachi A: Effects of lysine to arginine mutations in HIV-1 Vif on its expression and viral infectivity. Int J Mol Med 18: 679-683, 2006

15. Kao S, Khan MA, Miyagi E, Plishka R, Buckler-White A and Strebel K: The human immunodeficiency virus type 1 Vif protein reduces intracellular expression and inhibits packaging of APOBEC3G (CEM15), a cellular inhibitor of virus infectivity. J Virol 77: 11398-11407, 2003.

16. Karczewski MK and Strebel K: Cytoskeleton association and virion incorporation of the human immunodeficiency virus type 1 Vif protein. J Virol 70: 494-507, 1996.
17. Lebkowski JS, Clancy S and Calos MP: Simian virus 40 replication in adenovirus-trandformed human cells antagonizes gene expression. Nature 317: 169-171, 1985.

18. Kimpton, J and Emerman M: Detection of replication-competent and pseudotyped human immunodeficiency virus with a sensitive cell line on the basis of activation of an integrated $\beta$-galactosidase gene. J Virol 66: 2232-2239, 1992.

19. Willey RL, Smith DH, Lasky LA, Theodore TS, Earl PL, Moss B, Capon DJ and Martin MA: In vitro mutagenesis identifies a region within the envelope gene of the human immunodeficiency virus that is critical for infectivity. J Virol 62: 139-147, 1988.

20. Laemmli UK: Cleavage of structural proteins during the assembly of the head of bacteriophage T4. Nature 227: 680-685, 1970.

21. Fujita M, Akari H, Sakurai A, Yoshida A, Chiba T, Tanaka K, Strebel K and Adachi A: Expression of HIV-1 accessory protein Vif is controlled uniquely to be low and optimal by proteasome degradation. Microbes Infect 6: 791-798, 2004

22. Izumi T, Takaori-Kondo A, Shirakawa K, Higashitsuji H, Itoh K, Io K, Matsui M, Iwai K, Kondoh H, Sato T, Tomonaga M, Ikeda S, Akari H, Koyanagi Y, Fujita J and Uchiyama T: MDM2 is a novel E3 ligase for HIV-1 Vif. Retrovirology 6: 1, 2009. 